\title{
DOUBLE MUTATION CARRIER IN BRCA2 AND MUTYH GENES: A CASE REPORT IN A YOUNG BREAST CANCER PATIENT
}

Deidimar C.B. Abreu'1, Leandro G. Oliveira', Pedro P. B. Abreu', Vera A. Saddi¹

IInstituto Goiano de Oncologia e Hematologia, Pontifícia Universidade Católica de Goiás, Associação de Combate ao Câncer em Goiás - Goiânia (GO), Brazil.

Objectives: To describe a case of a young female patient (32 years old) diagnosed with breast cancer and submitted to a genetic testing with an unusual result. Case description: The tumor was an invasive ductal carcinoma, estrogen and progesterone receptors positive and HER2 negative. The family history revealed a paternal aunt with bilateral breast cancer at about 60 years old, a paternal grand uncle with a central nervous system cancer, and another paternal granduncle with leukemia, who had three daughters with breast cancer at young age. During the treatment period, the father of the patient was also diagnosed with breast cancer. The genetic test revealed a concomitant double pathogenic variant, namely c.9382C>T (p.Arg3128*) in BRCA2 and c.1187G>A (p.Gly396Asp) in the MUTYH gene. Discussion: A pathogenic mutation in BRCA2 gene is associated to increased risk of developing breast and ovary cancer in young age, compared to general population. The risk of pancreatic cancer and melanoma are also increased by this mutation. A pathogenic mutation in the monoallelic MUTYH gene seems to slightly increase the risk of developing colorectal cancer, compared to average woman. Although the cancer risk in individuals who carry multiple pathogenic variants has not been established for combined MUTYH and BRCA2 pathogenic variants, the identification of multiple pathogenic variants does allow for screening for cancers associated with both syndromes and has implications for cancer risk for family members. More information is needed on the frequency of multiple pathogenic variants, as well as the phenotypic spectrum when multiple pathogenic variants are present. 